\title{
Lesiones de la Copa de Europa de Atletismo
}

\author{
María C. Martín Fernández ${ }^{a}$, Consuelo Sánchez Arjona a , Carlos Melero Romeroa ${ }^{\text {, Ana Navarro Sanz }}$, \\ Yolanda Ruiz Martínez ${ }^{\text {y }}$ Juan Elena Gamboa ${ }^{a}$
}

${ }^{a}$ Centro Andaluz Medicina del Deporte (CAMD). Málaga.

'Fundación Deportiva Ayuntamiento de Málaga. Málaga.

\section{RESUMEN}

Introducción y objetivos: La Copa de Europa se debe a Bruno Zauli. Este año se celebró en Málaga. Conocer las patologías producidas y su frecuencia.

Métodos: Se realizó un estudio estadístico, descriptivo, prospectivo. Se utilizó una historia clínica, el programa Excel y una búsqueda bibliográfica.

Resultados: Durante estos días tuvieron lugar 23 intervenciones: 10 atletas y 13 no deportistas. Según su rol: el 18,59\% eran jueces; el 24,79\% organización; el 8,68\% prensa, y el $47,94 \%$ atletas. Por sexos: el $56,52 \%$ varones, y el $43,48 \%$ mujeres. Por países: el $43,38 \%$ españoles; el $18,9 \%$ italianos; el 9,68\% británicos; el 9,68\% rusos; el 9,68\% rumanos; el 4,34\% ucranianos, y el 4,34\% finlandeses. Los motivos de consulta: el $48,68 \%$ dolor; el $38,32 \%$ heridas; el $9 \%$ lipotimias, y el $4 \%$ picaduras de insectos. Los diagnósticos fueron: el $40 \%$ lesiones musculosqueléticas; el 38,32\% heridas; el $9 \%$ golpe de calor; el 4,34\% gastroenteritis; el 4,34\% cefalea, y el 4 picadura de insecto. Tratamientos: crioterapia en el $26,08 \%$; fisioterapia en el $4,34 \%$; vendaje compresivo en el $4,34 \%$; farmacoterapia en el $34,78 \%$; curas en el $47,82 \%$, e hidratación más crioterapia en el $8,69 \%$.

Conclusiones: En estos eventos hubo baja demanda asistencial; más frecuente la consulta durante la competición; significación del golpe de calor en las personas procedentes del este de Europa; la patología más común ha sido la musculosquelética; los traslados al hospital son escasos y derivados de éstas. En conclusión, se puede decir que el nivel de asistencia sanitaria para deportistas y no deportistas parece ser adecuado para cubrir las necesidades presentadas.

PALABRAS CLAVE: Atletismo. Copa de Europa. Patologías. Asistencia sanitaria.

\section{ABSTRACT}

Introduction and objectives: The European Cup was created by Bruno Zauli. This year the competition was held in Malaga (Spain). Our objective was to determine the health problems produced and their frequency.

Methods: A descriptive, prospective study was performed. Clinical history, the Excel program, and a literature search were used.

Results: Twenty-three patients were treated: 10 athletes and 13 non-athletes. According to role, $18.59 \%$ were field judges, $24.79 \%$ worked in organization, $8.68 \%$ were reporters, and $47.94 \%$ were athletes. By sex, $56.52 \%$ were men and $43.48 \%$ were women. Country of origin was Spain in $43.38 \%$, Italy in $18.9 \%$, Britain in $9.68 \%$, Russia in $9.68 \%$, Romania in $9.68 \%$, Ukrain in $4.34 \%$, and Finland in $4.34 \%$. Reasons for consultation were pain in $46.68 \%$, injuries in $38.22 \%$, dizziness in $9 \%$, and insect bites in $4 \%$. Diagnosis was muscle-skeleton injuries in $40 \%$, injuries in $38.32 \%$, sunstroke in $9 \%$, headache in $4.34 \%$, gastroenteritis in $4.34 \%$, and insect bites in $4 \%$. Treatments consisted of cryotherapy in $26.08 \%$, physiotherapy in $4.34 \%$, compression bandaging in $4.34 \%$, pharmacotherapy in $34.78 \%$, topical treatments in $47.82 \%$, and water therapy in $8.69 \%$.

Conclusions: Demand for healthcare at these events is low; consultation is more frequent during the competition. Sunstroke is frequent in athletes from eastern Europe. The most common diagnosis was musculoskeletal injuries. Most transfers to hospital were for this reason, although the number of transfers was small. In conclusion, healthcare provision to athletes and non-athletes was sufficient to meet requirements.

KEY WORDS: Athletics. European cup. Health problems. Consultations. 


\section{INTRODUCCIÓN}

La Copa de Europa de Atletismo hay que atribuírsela a Bruno Zauli. La primera vez que tuvo carácter oficial fue en París, en mayo de 1964. En las 2 primeras décadas se disputaban rondas preliminares. En 1983 se decidió cambiar para que todos los países pudieran competir en un mismo fin de semana, la final pasó a llamarse "Superliga", donde competían los 8 mejores equipos femeninos y masculinos ${ }^{1}$.

Este año se ha realizado en Málaga, en el Estadio de Atletismo "Ciudad de Málaga" los días 28 y 29 de junio de 2006. En los días previos se realizaron los entrenamientos en el Polideportivo de Carranque y en el propio Estadio (aquí sólo para disco, peso y martillo) jornadas de mañana y tarde, y solamente por la mañana los días de la competición. El horario de entrenamiento durante los 2 primeros días fue de 11 a 13 y de 17 a 20 horas y los otros de 10 a 12. La competición se realizó de 18 a 22 horas.

Las pruebas de atletismo que tuvieron lugar fueron: lanzamientos de martillo, peso, disco y pértiga; saltos de altura y longitud; triple salto; $400 \mathrm{~m}$ vallas; carreras de 100, 400, 800, $1.500,3.000$ y 5.000 ; 3.000 obstáculos; $4 \times 100$; $4 \times 400$, todas realizadas por varones y mujeres; $110 \mathrm{~m}$ vallas sólo para varones y $100 \mathrm{~m}$ vallas para mujeres ${ }^{2}$.

Uno de los grandes problemas a los que se enfrentan sus propios organizadores es calcular la previsión de la demanda médica asistencial derivada de grandes acontecimientos deportivos y sociales con gran poder de convocatoria ${ }^{3-10}$, ya que las necesidades de este terreno son variables y relativamente impredecibles ${ }^{11}$. Esto, junto a la escasa bibliografía acerca del tema nos llevó a plantear el estudio acerca de las lesiones atendidas en la Copa de Europa de Atletismo 2006. El objetivo del estudio fue conocer el número de patologías que se producen durante este tipo de eventos y cuáles son las que se presentan con más frecuencia.

\section{MÉtodos}

La Copa de Europa de Atletismo ha sido organizada por el Ayuntamiento de Málaga, sobre todo, por su médico del deporte, la Real Federación Española de Atletismo y la Junta de Andalucía.

Una clínica médica en el estadio y en el polideportivo se ha hecho cargo de todas las patologías surgidas durante los entrenamientos y la competición. La asistencia sanitaria se ha llevado a cabo, fundamentalmente, por el personal sanitario del Ayuntamiento de Málaga y del Centro Andaluz de Medicina del Deporte de la misma ciudad, los cuales han atendido las lesiones acaecidas durante el evento. Se contrató un servicio de ambulancia y UVI-móvil con la Clínica El Rincón, con su personal.

Se realizó un estudio estadístico, descriptivo y prospectivo de las lesiones ocurridas en la Copa de Europa de Atletismo. Para ello se utilizó una historia clínica con los siguientes datos: fecha, nombre, edad, sexo, nacionalidad, función dentro del campeonato (atleta, juez, personal de la organización, personal de prensa, espectador), cuándo se trató (competición o entrenamiento), dónde (Polideportivo de Carranque o Estadio de Atletismo), motivo de consulta, zona de la lesión, diagnóstico, tratamiento, si se realizó traslado, medio de traslado y lugar al que se trasladó. Los países participantes fueron, para los varones: Alemania, Italia, Polonia, España, Rusia, Francia, Gran Bretaña, Irlanda del Norte, Finlandia y Ucrania. Para las mujeres: Polonia, Suecia, Alemania, España, Rumania, Francia, Ucrania, Gran Bretaña, Irlanda del Norte y Rusia.

Para el estudio estadístico se utilizaron los programas Excel y Word. Además de realizar una búsqueda bibliográfica en Pubmed y libros de texto.

\section{Resultados}

Durante los 4 días tuvieron lugar 23 intervenciones, de las cuales 10 fueron de atletas y 13 de no deportistas. Todas fueron en el Estadio de Atletismo. Tres actuaciones fueron durante los entrenamientos (una el día 27 y 2 el 29). Las intervenciones durante la competición fueron 20 ( 14 el primer día y 6 el segundo). Sólo hubo 2 traslados: el día 27 de una lesión traumatológica del pie y el día 29 de un atleta con lesión en la columna vertebral. Ambas se derivaron a la Clínica El Ángel, y el traslado se realizó por medio de UVI-móvil para realización de pruebas complementarias. También se realizó una ecografía por una posible rotura muscular a una atleta, esta prueba tuvo lugar en la consulta instalada en el mismo estadio.

Dividimos las intervenciones según su función dentro del campeonato: el $18,59 \%$ a jueces; el $24,79 \%$ a personal de la organización; el 8,68\% a personal de prensa, y el $47,94 \%$ a atletas (fig. 1).

Si la descripción se realiza por sexos: el 56,52\% fueron varones (el 18,59\% jueces, el 21,94\% atletas, el 7,41\% organización y el $8,68 \%$ prensa), y el $43,48 \%$ mujeres (el $17,38 \%$ organización y el 26,08\% atletas) (fig. 2).

Por países: el $43,38 \%$ españoles (el 18,59\% jueces y el $24,79 \%$ organización); el $18,90 \%$ italianos (el 10,22\% atletas y el $8,68 \%$ personal de prensa); el 9,68\% británicos (todos 
TRABAJOS ORIGINALES

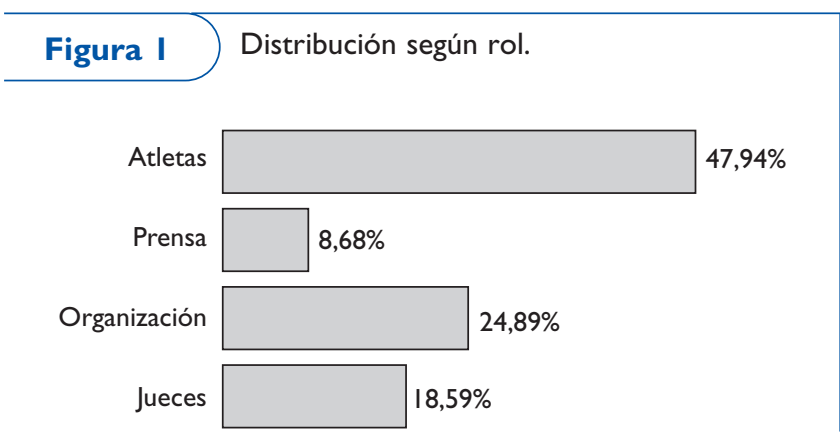

\section{Figura $2 \int$ Distribución según sexos.}

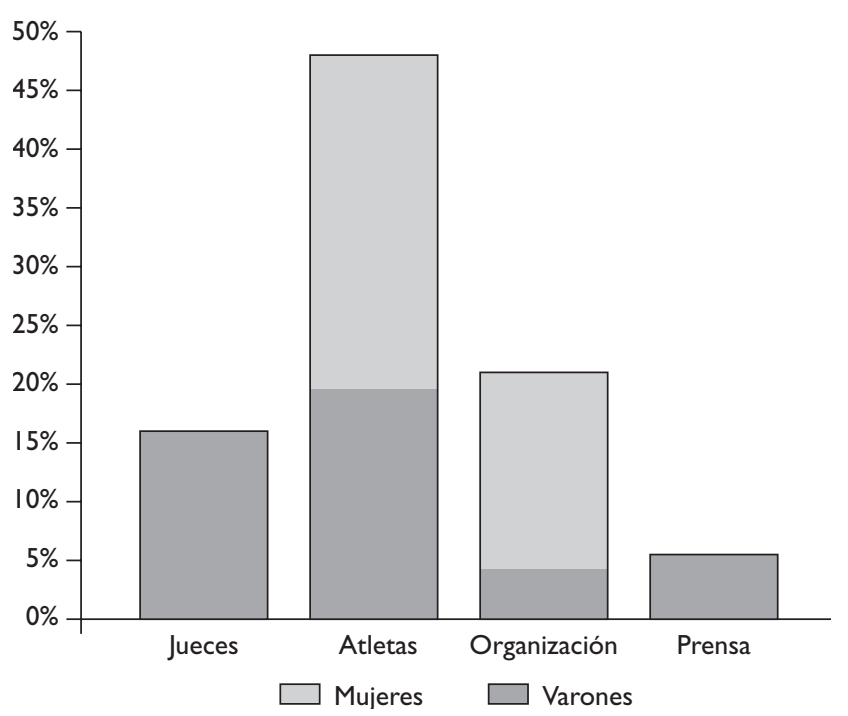

atletas); el 9,68\% rusos (todos atletas); el 9,68\% rumanos (todos atletas); el 4,34\% ucranianos (que era un atleta), y el $4,34 \%$ finlandeses (también atleta) (fig. 3).

Los motivos de consulta fueron: el $48,68 \%$ por dolor; el $4 \%$ por picaduras de insectos; el $38,32 \%$ por heridas, y el $9 \%$ por lipotimias (fig. 4).

Se presenta una distribución de los juicios clínicos según el carácter de deportista y no deportista del paciente, lo que nos permite conocer las patologías más frecuentes en uno u otro colectivo (tabla I).

Los tratamientos que se dieron fueron: crioterapia en el 26,08\%; fisioterapia en el 4,34\%; vendaje compresivo en el 4,34\%; farmacoterapia en el $34,78 \%$; curas en el $47,82 \%$, e hidratación más crioterapia en el 8,69\% (fig. 5). Las zonas de las lesiones fueron: espalda el 4,34\%; mano el 13,04\%; muslo el $13,04 \%$; rodilla el $8,69 \%$; tobillos el $8,69 \%$, y cara el $4,34 \%$.

\section{Figura 3 Distribución según países.}


Figura 5 Tratamientos.

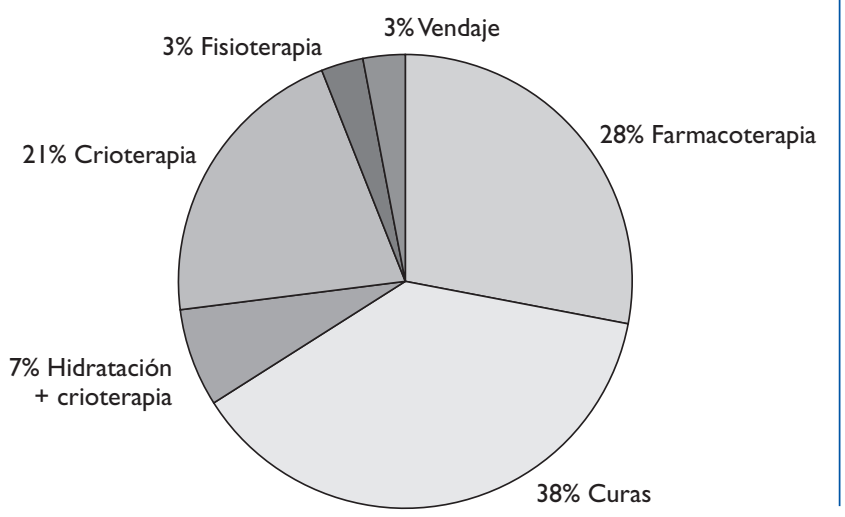




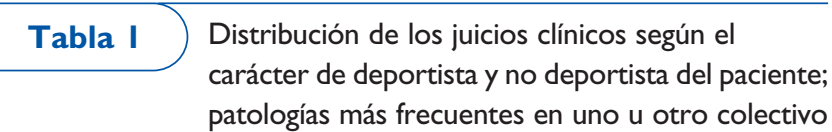

\begin{tabular}{|lccc|}
\hline Juicio clínico & Total & Deportistas & $\begin{array}{c}\text { No } \\
\text { deportistas }\end{array}$ \\
\hline $\begin{array}{l}\text { Musculosqueléticas } \\
\text { (contusiones, esguinces, } \\
\text { tendinitis, rotura muscular) } \\
\text { (\%) }\end{array}$ & 40 & 21,18 & 18,82 \\
\hline Heridas (\%) & 38,32 & 17,76 & 20,56 \\
\hline Golpe de calor (\%) & 9 & 9 & 0 \\
\hline Picadura de insecto (\%) & 4 & 0 & 4 \\
\hline Cefaleas (\%) & 4,34 & 0 & 4,34 \\
\hline Gastroenteritis (\%) & 4,34 & 0 & 4,34 \\
\hline Total (\%) & 100 & 47,94 & 52,06 \\
\hline
\end{tabular}

\section{Discusión}

Al revisar la literatura médica se pone de manifiesto la escasa información que hay acerca de la asistencia médica en grandes acontecimientos deportivos.

Nuestro trabajo muestra la demanda asistencial de la Copa de Europa de Atletismo, que ha sido baja y de poca gravedad. La atención médica fue más frecuente en varones, aunque las deportistas femeninas se lesionaron más que los atletas masculinos. Asistimos a menos atletas que a personal no deportista, al igual que en la Olimpiada de Barcelona, los Juegos Olímpicos de Invierno de $2002^{12}$ y los Juegos Paralímpicos de Invierno de $2002^{13}$, y a diferencia que en los Juegos del Mediterráneo ${ }^{14}$.
Las lesiones traumáticas que sobrevienen en la práctica deportiva son de producción instantánea y de cierta grave$\mathrm{dad}^{2}$. Pueden incidir sobre el hueso o sobre cualquiera de los tejidos blandos. Así, en el hueso se pueden encontrar fracturas y/o luxaciones; mientras que en el tejido blando pueden producirse las siguientes lesiones: contusiones, erosiones, contracturas, elongaciones, distensiones, desgarros, tendinitis y rupturas. $\mathrm{Al}$ analizar nuestros resultados, se encontró que lo más frecuente fueron las patologías relacionadas con el aparato locomotor, al igual que en los demás estudios ${ }^{12-14}$. De ellas, las más frecuentes han sido patologías del tejido blando. También los 2 traslados hospitalarios se derivaron de éstas, lo que está en concordancia con el resto de estudios encontra$\operatorname{dos}^{3,4,11-14}$.

Las heridas fueron la segunda patología más diagnosticada. Merece la pena destacar que las produjeron 2 orígenes totalmente distintos, que dependían de a quién afectaran. Así, hubieron heridas por abrasiones en la pista en las caídas de los deportistas y las surgidas por rozaduras del calzado debidas al calor de los no deportistas.

El golpe de calor se precipita por una producción excesiva de calor endógeno. Suele afectar a jóvenes no entrenados o poco aclimatados, como consecuencia de la realización de un ejercicio intenso. Para su aparición es necesario: temperaturas ambientales elevadas, humedad elevada y/o escasa velocidad del viento ${ }^{15}$. En nuestro caso fue la tercera patología más frecuente, y se debió a la falta de climatización del deportista (se produjo en los que procedían del este de Europa, poco habituados a altas temperaturas), a la excesiva humedad y a las altas temperaturas para ellos.

\section{Bibliografía}

1. Disponible en: www.rfea.es/competi/malaga2006/eng/eng_history.htm (consultada el 3 de julio de 2006).

2. Pastrana R, et al. Lesiones deportivas y rehabilitación. Monografías de Rehabilitación. Vol. 1. Madrid: Ferjisa Artes Gráficas; 1988. p. 13-7.

3. Baker WM, Simone BM, Nieman JT, Daly A. Special event medical care: the 1984 Los Angeles Summer Olimpyc Experience. Ann Emerg Med. 1986;15:185-90.
4. Thompson JM, Savoia G, Powell G, Challis EB, Law P. Level of medical care requiered for mass gatherings: the XV Winter Olimpyc Games in Calgary, Canada. Ann Emerg Med. 1991;20: 385-90.

5. Wat J, Bottomley MB, Read MT. Olimpyc athletics medical experience, Seoul-personal views. Br J Sports Med. 1989;23:76-9.

6. Wolfe SM, Behrman M. Heat stoke and community rus. Br Med J. 1981;282:2060. 
7. Levens LK, Durhan JE. Pop-music festivals: somemedical aspects. Br Med J. 1971;1:218-20.

8. Schlicht J, Mitcheson M, Henry M. Medical aspects of large outdoor festivals. Lancet. 1972;1:948-52.

9. Yaquub BA, Al-Harthi SS, AL-Orainey IO, Laajam MA, Obeid MT. Heat stroke at Mekkah pilgrimage: clinical characteristics and course of 30 patients. Q J Med. 1986;59:523-30.

10. Chapman KR, Carmichael FJ, Goode JE. Medical services for outdoor rock music festivals. Can Med Assoc J. 1982;126: 935-8.

11. Garcés JM, Gutiérrez-Cebollada J, Benito P, Estrada J, Torres X, Molina L, et al. Una experiencia única: los Juegos Olímpicos de Barcelona 1992. Med Clin (Barc). 1996;106:290-3.
12. Grissom CK, Finnoff JT, Murdock DC, Culberson JT. Nordic venue medical services during the 2002 Winter Olympics. J Emerg Med. 2006;30:203-10.

13. Webborn N, Willick S, Reeser JC. Injuries among disabled athletes during the 2002 winter Paralympic Games. Med Sci Sports Exerc. 2006;38:811-5.

14. Adamuz Ruiz MC, Prada Pérez A, Ordóñez Muñoz FJ, Rosety Rodríguez M. Organización, planificación, gestión y resultados de la asistencia sanitaria en los XV Juegos Mediterráneos de Almería 2005. Revista MD. 2005;3:33-42.

15. Ballesteros R. Patología asociada al calor. Traumatología y Medicina Deportiva. Medicina del deporte. Madrid: Paraninfo; 2002. p. 245-8. 